$\Rightarrow$ LUNG CANCER

\title{
Reliable results from several DNA sources
}

We show the
simplicity of
use of a source
of biological
material ...
to obtain
clinically
relevant
genomic
information

Genetic testing facilitates the management of patients with lung cancer, who can receive targeted therapies if actionable mutations are detected. Tumour biopsy specimens are the most common source of genetic material for those analyses, but sometimes the procedure cannot be performed or the quality of the samples obtained is not optimal. In addition, lung biopsy sampling is an invasive procedure that carries risks, which can sometimes be life threatening. Now, two recent studies describe optimized procedures to analyse circulating cell-free DNA (cfDNA) in liquid biopsies or DNA in centrifuged supernatants from fine-needle aspirate (FNA) samples.

One of the initiatives from the Actionable Genome Consortium was the development and validation of a novel next-generation sequencing (NGS) assay for analysis of plasma liquid biopsy samples termed 'ultra-deep NGS. "This assay enables a sequencing depth of up to $50,000 \times$ and incorporates an algorithm to filter white-blood-cell sequences, to ensure sensitivity and specificity," explains lead author Robert Li.

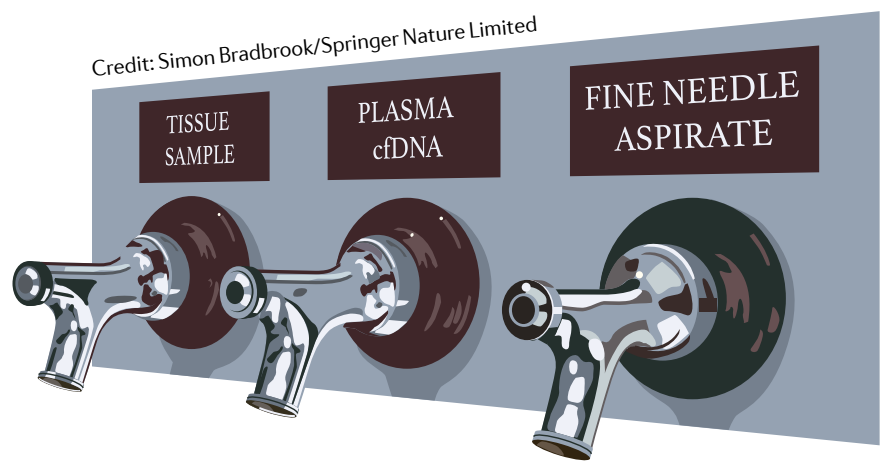

The assay was evaluated in samples from patients with non-small-cell lung cancer (NSCLC) for whom information for tumour tissue genotyping was also available, the results of which were used to define three subgroups. Patients were assigned to the driver-positive $(n=91)$ or driver-negative $(n=19)$ groups on the basis of the presence or absence of alterations in 8 pre-specified oncogenes, or to the driver-unknown group $(n=17)$ when tissue biopsy material was unavailable or not suitable for DNA analysis. Identical driver variants in cfDNA were identified in 68 patients (75\%) in the driver-positive group, in none of the patients in the driver-negative group and in 4 patients in the driver-unknown group. Mutations in EGFR that confer resistance to targeted therapies were assessed in tissue samples from 23 patients, and findings were concordant with cfDNA samples in 19 of them (83\%).

Assessment of potential confounding effects was performed by grouping samples according to the time between blood draw and biopsy and according to the therapy received during that period of time. No statistically significant differences were detected.

In some patients, the amount of detectable cfDNA in plasma is limited. Several studies have demonstrated that centrifuged supernatants from FNA needle rinses (otherwise discarded) can be used for molecular testing. In a prospective study, FNA supernatants were obtained from consecutive patients with lung cancer $(n=150)$, and 104 of $116(90 \%)$ samples were successfully sequenced by NGS. Of those,
35 samples had a concurrent tissue specimen from the same lesion with NGS results available, 34 of which were concordant (97\%).

Plasma samples from the same patient were available for 45 of the 104 FNA samples, 40 of which had been collected within 3 months. Concordance was observed in $84 \%$ of paired samples in which mutations in the same driver genes had been analysed. Importantly, 4 mutations were detected in the FNA supernatant sample but not in plasma, as confirmed in paired tissue specimens.

"We show the simplicity of use of a source of biological material (that would be otherwise discarded) to obtain clinically relevant genomic information," describes Sinchita RoyChowdhuri. "This is importantly for patients from whom we have insufficient tissue for molecular testing, and who would require a repeat biopsy to obtain tissue for genotyping," she adds.

Both Li and Roy-Chowdhuri are optimistic about the technical implications of their results. "We need more prospective clinical studies to generate evidence that could lead to the incorporation of plasma NGS into practice guidelines," opines Li. "The technologies used in this study can be further optimized for the detection of early stage cancers and minimal residual disease," he adds. "Our goal is to incorporate the detection of RNA fusions in these assays," Roy-Chowdhuri explains, concluding, "We hope to raise awareness in the oncology community about the utility of FNA supernatants for genotyping."

Diana Romero

ORIGINAL ARTICLES Li, B. T. et al. Ultra-deep next-generation sequencing of plasma cell-free DNA in patients with advanced lung cancers: results from the Actionable Genome Consortium Ann. Oncol.https://doi.org/10.1093/annonc/ mdz046 (2019)| Hannigan, B. et al. Liquid biopsy assay for lung carcinoma using centrifuged supernatants from fine needle aspiration specimens. Ann. Oncol. https://doi.org/10.1093/ annonc/mdz102 (2019) 